\title{
OVERVIEW OF COLLIMATION IN THE NEXT LINEAR COLLIDER
}

\author{
P. Tenenbaum, P. Emma, L. Keller, Y. Nosochkov, T. Raubenheimer, M. Woodley, SLAC *
}

\begin{abstract}
The Next Linear Collider (NLC) requires a substantial system of beam collimators to minimize the halo-related backgrounds at the interaction point, provide machine protection against errant pulses, and limit radiation doses throughout the accelerator. In particular, the NLC design contains a complete 5-dimensional ( $\mathrm{x}, \mathrm{x}^{\prime}, \mathrm{y}, \mathrm{y}^{\prime}$, energy) collimation system at the entrance and exit of the main linac; transverse collimators at the electron and positron sources, at the $250 \mathrm{MeV}$ point in the injector linac, and immediately upstream of the damping rings; a series of collimators at points with substantial z-energy correlation for longitudinal collimation; and a final set of transverse and energy collimators in the final focus, a few hundred meters from the IP. We describe the systems above and estimate the intensity of the halo at each point, as well as the efficiency with which the halo is removed by the collimators.
\end{abstract}

\section{INTRODUCTION}

The Next Linear Collider (NLC) will require in excess of 10 megawatts per beam to achieve its luminosity goals at 1 $\mathrm{TeV}$ CM. This implies that each linac pulse will accelerate over $10^{12}$ particles with $120 \mathrm{~Hz}$ linac pulse rate. Such parameters present a substantial challenge to beam-handling technology and techniques.

Many of the beam-handling challenges of the NLC will be addressed through careful beam collimation. Collimation in the NLC will be used to remove large-amplitude particles which would potentially cause backgrounds in the detectors; to protect the detector and accelerator components from damage by mis-steered or off-energy beams; and to limit radiation doses throughout the accelerator by providing well-shielded locations where beam tails can be removed ("concrete closets"). The collimation systems which accomplish these goals are located throughout the facility, as shown in Figure 1.

\section{POST-LINAC COLLIMATION}

Figure 2 shows the optical functions of the post-linac collimation system [1]. The post-linac collimation system's primary requirements are to eliminate particles which can cause unacceptable backgrounds in the detector and to provide machine protection for the detector from bunch trains with large energy or betatron excursions.

Because the NLC main linac is pulsed, it is conceivable that energy errors will be frequent and will occur with no precursor. This implies that off-energy beams which reach

\footnotetext{
* Work supported by U.S. Department of Energy, Contract DE-AC03-
} $76 \mathrm{SF} 00515$.

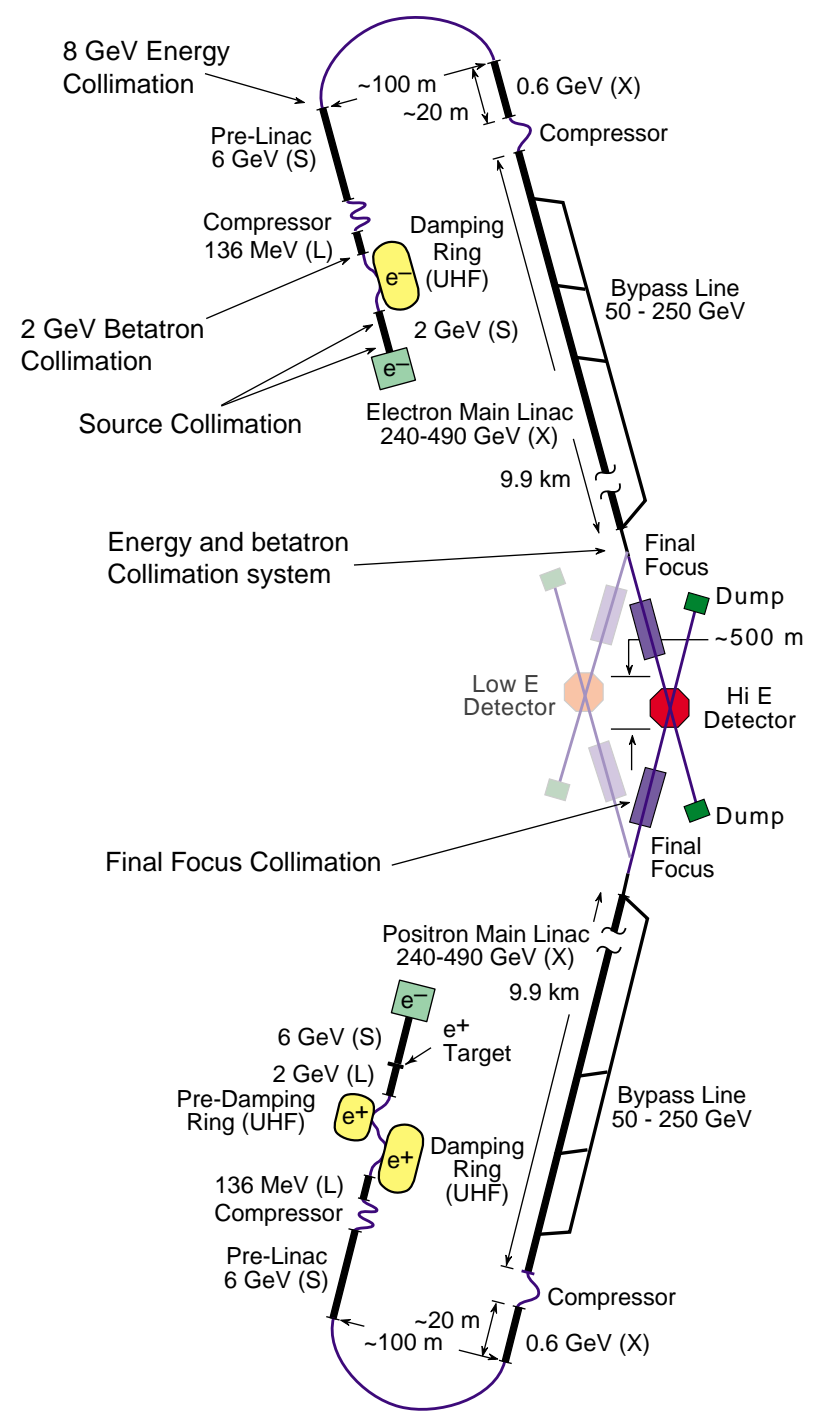

Figure 1: Locations of collimation systems in the NLC.

the end of the linac may occur frequently. By contrast, all systems which might cause a large betatron oscillation have been designed to be incapable of rapid changes of state; changes that could drive a beam out of the collimation aperture will require more than the $8 \mathrm{msec}$ inter-pulse period, and thus there will be a precursor on a previous pulse. The machine protection system will be designed to detect the precursor to an errant pulse and abort the next beam before damping ring extraction. Therefore, it is expected that bunch trains with unacceptable betatron oscillations will rarely reach the collimation system. The combination of these factors suggests that the energy collimation system at the end of the linac should be far more resistant to damage from beam power than the betatron collimation system. 


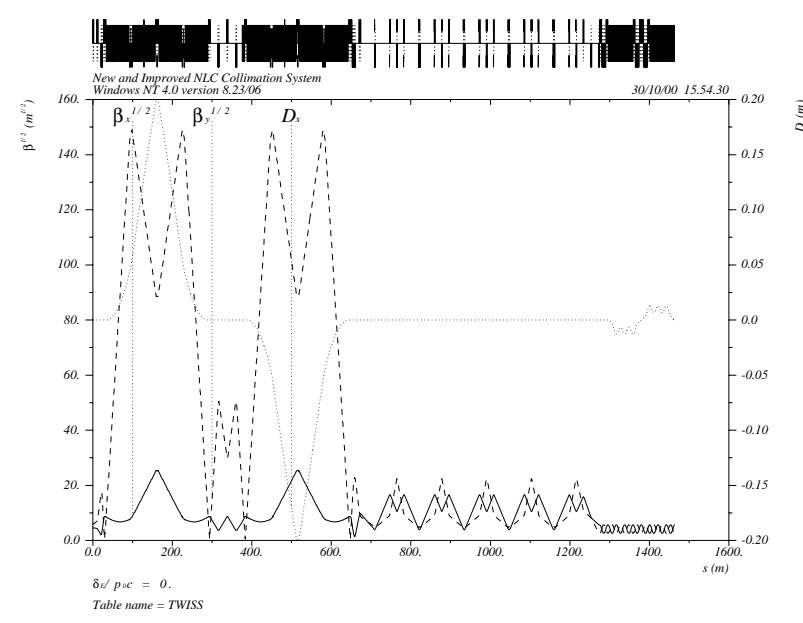

Figure 2: Optical functions of the NLC post-linac collimation system.

The design in Figure 2 accomplishes this by placing the energy collimator in front of the betatron collimator, by using large $\beta_{y}$ and $\eta_{x}$ values at the energy collimators to ensure that the beam is too large to cause collimator damage, and by the use of "consumable" betatron collimators that can tolerate a certain amount of beam-induced damage without unacceptable deterioration in their performance.

The post-linac collimation system is designed to provide redundant collimation: each degree of freedom $\left(x, x^{\prime}, y, y^{\prime}, \delta\right)$ is collimated twice. This design requires a substantial number of collimators, each of which generates beam jitter amplification through transverse wakefields. The energy collimation system is potentially the worst source of such jitter, because the desired energy aperture $( \pm 1 \%)$ is close to the expected RMS energy jitter $(0.22 \%)$. The primary wakefield effect of the energy collimators - coupling of energy jitter into the horizontal betatron degree of freedom - is ameliorated by use of optical symmetries which cause the energy-to-betatron jitter coupling to cancel. No such symmetries were available for the betatron collimation system, and analytic estimates had indicated that the degree of jitter amplification in the vertical plane would be unacceptable. Recent analytic results [2], experimental measurements [3], and MAFIA simulations [4] indicate that the wakefields may be less severe than originally expected.

The collimator apertures in the post-linac system are designed to stop particles which would otherwise shower the vertex detector with synchrotron radiation emitted in the final doublet quadrupoles [5]. The apertures correspond to approximately $\pm 5 \sigma_{x}, \pm 30 \sigma_{y}$, and $\pm 1 \%$ in energy. The number of particles which may be stopped on each linac pulse is determined by the flux of muons that are generated in the collimators and reach the detector. Present estimates indicate that $10^{9}$ particles may be stopped per linac pulse (out of $10^{12}$, or $0.1 \%$ ) without causing unacceptable muon backgrounds in the detector. This is many orders of magnitude larger than the expected beam halo population at the end of the linac, but is consistent with SLC experience [5]. The system's transmission of halo particles is approximately $10^{-5}$, implying that up to $10^{4}$ halo particles might pass through the collimation system into the final focus. These particles are absorbed by additional collimators in the high- $\beta$ regions of the final focus.

\section{PRE-LINAC COLLIMATION}

The experience of the SLC indicated that most of the beam halo was generated at the low-energy end of the accelerator, in the damping rings and bunch compressors. If such a situation proves to be the case in the NLC as well, it is sensible to collimate the halo at as low an energy as possible: the amount of beam power which must be absorbed is low, and the machine protection requirements are eased by the larger geometric emittance of the beam.

The present design of the NLC injector separates the collimation functions of the low-energy beam. Energy collimation is performed at the high-energy end of the injector, just upstream of the turnaround of the second bunch compressor. This provides protection against off-energy beams from the $6 \mathrm{GeV}$ linac which separates the damping ring from the turnaround. The energy slits achieve protection against errant bunch trains in a similar fashion to the postlinac energy collimator, but the low beam energy permits smaller $\beta_{y}$ and larger $\eta_{x}$ to be used [6]. Figure 3 shows the optical functions of the pre-linac energy collimation system.

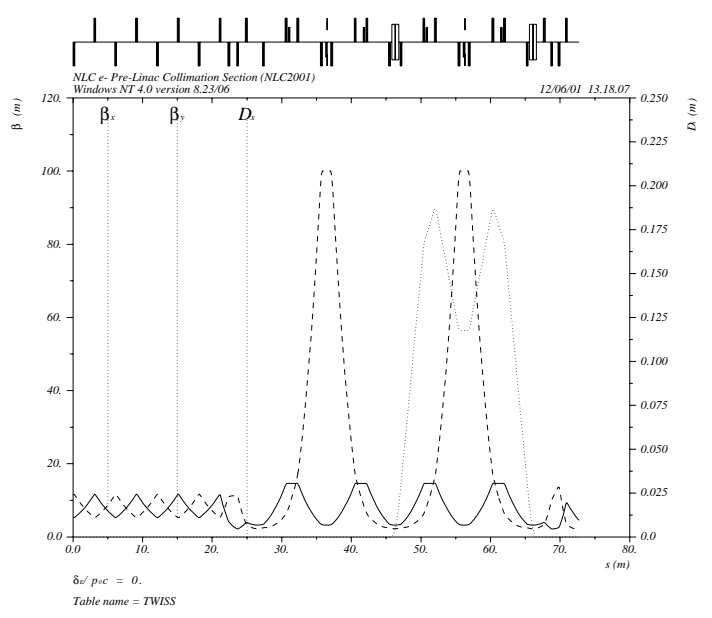

Figure 3: Optical functions of the NLC pre-linac energy collimation system.

Pre-linac betatron collimation is performed immediately downstream of the damping ring extraction, where the beam energy is $1.98 \mathrm{GeV}$. This allows the expected damping ring halo to be eliminated at the lowest energy possible, and provides optimal protection against beams mis-steered by extraction transients. It is also advantageous from a beam dynamics standpoint: since the collimation region requires relatively large $\beta$ functions to achieve machine protection, it will have a large chromaticity and small bandwidth; by placing the betatron collimation system at the 
damping ring extraction, where the RMS energy spread and energy jitter are both small $(0.09 \%$ and $0.1 \%$, respectively), a highly chromatic system can be used without chromatic correction. At this time a final design of the optics is not available. The optics is likely to be similar to the postlinac betatron collimation system, and will achieve a halo transmission of $0.1 \%$ or better. Based on beam transport studies of the NLC main linac, the aperture of the pre-linac system, in beam sigmas, will probably need to be slightly tighter than that of the post-linac system [7].

\section{INJECTOR COLLIMATION}

Upstream of the main damping ring, the charge density of the beam is much lower and machine protection is less of a driving issue. Furthermore, the phase space of the beam which emerges from the damping ring is almost entirely determined by the ring dynamics, so collimators upstream of the damping ring do not improve the halo observed at ring extraction. The principal purpose of collimators upstream of the damping ring is radiation control. The SLC damping ring complex suffered severe and ongoing deterioration from continual exposure to high levels of radiation. The NLC beams have 35 times as much average current as the SLC, and a corresponding increase in uncontrolled radiation exposure in the NLC's sources, booster linacs, and damping ring complexes is unacceptable. The principal focus of the injector collimation systems is to confine the anticipated beam losses to areas which can be heavily shielded, and thus protect the rest of the accelerator from such high radiation doses.

Collimation upstream of the damping rings will be performed at the injection point to the rings, at the end of the beam-generation systems where the beam energy is 250 $\mathrm{MeV}$ and the beams are fully bunched, and at the sources where the beams remain non-relativistic.

\section{BUNCH LENGTH COLLIMATION}

One of the possible sources of beam halo in the SLC was non-Gaussian longitudinal tails. A particle far from the longitudinal core of the beam could be severely impacted by wakefields, and would also end up extremely far from the design energy at the end of the linac. The SLC had a substantial low-energy tail which accompanied the beam, and this was ameliorated by shaping the longitudinal distribution of the beam [8]. One means to avoid such a source of halo in the NLC is to collimate the longitudinal tail; this can only be accomplished by introducing a $z-\delta$ correlation and a simultaneous $x-\delta$ correlation (ie, dispersion), and then introducing a horizontal collimator. Because the NLC has two bunch compressors downstream of the damping ring, the collimation must be performed at a location where the longitudinal phase is the same as in the main linac.

The present design incorporates such collimators at one location in the first bunch compressor and two locations in the second bunch compressor; a final longitudinal cut is performed by the energy collimation portion of the postlinac system, where the longitudinal tails of the beam have been driven off-energy by the main linac RF. Studies indicate that the collimators can reduce a longitudinal damping ring halo to one part in 500 of its original intensity. The surviving halo particles occupy islands of stability in Xband buckets adjacent to the beam core's. Such "satellite bunches" will likely have a very large emittance and poor orbit, and further studies in the removal of these bunches must be performed.

\section{CONCLUSIONS AND FUTURE DIRECTIONS}

The NLC uses a large array of beam collimation systems to control beam backgrounds in the detector, provide machine protection from mis-steered or off-energy beams, and protect the bulk of the accelerator from unacceptable radiation damage. The collimation system designs have been studied in detail, and appear to provide acceptable performance.

Recent developments in the design of the post-linac collimation system offer the possibility of further improvements in performance. These include the use of octupole doublets to compact the portion of the beam halo which is in phase with the final doublet, and integrating the high- $\beta$ portions of the collimation system more thoroughly with the final focus so that both systems can be chromatically corrected by one set of sextupoles [9].

\section{REFERENCES}

[1] NLC Post-Linac Collimation Task Force, LCC-Note-0052, http://www-project.slac.stanford.edu/lc/ilc/TechNotes /LCCNotes/lcc_notes_index.htm.

[2] G. Stupakov, "High-Frequency Impedance of Small-Angle Collimators," these proceedings.

[3] P. Tenenbaum et al, "Transverse Wakefields from Tapered Collimators: Measurements and Analysis," these proceedings.

[4] C.-K. Ng, T.O. Raubenheimer, P. Tenenbaum, "Numerical Calculations of Short-Range Wakefields of Collimators," these proceedings.

[5] S. Hertzbach, "SR Fans in NLC FF," Proceedings $B D-I R$ 2000, http://accelerator.dl.ac.uk/ap/bdir2000/ proceedings.htm.

[6] Y. Nosochkov, P. Raimondi, T. Raubenheimer, LCC-Note0057, http://www-project.slac.stanford.edu/lc/ilc/TechNotes /LCCNotes/lcc_notes_index.htm.

[7] P. Tenenbaum, T.O. Raubenheimer, M. Woodley, "Sources of Beam Halo in the Next Linear Collider Main Linac," these proceedings.

[8] T. Usher, private communication.

[9] P. Raimondi, A. Seryi, "Halo Reduction by Means of Non Linear Optical Elements in the NLC Final Focus System," these proceedings. 